\title{
ВмJ Global Health Bridging research integrity and global health epidemiology (BRIDGE) statement: guidelines for good epidemiological practice
}

\author{
Sandra Alba (D) , ${ }^{1}$ Kristien Verdonck, ${ }^{2}$ Annick Lenglet, ${ }^{3,4}$ Susan F Rumisha,, 6 \\ Martijn Wienia, ${ }^{7}$ Imre Teunissen, ${ }^{1}$ Masja Straetemans, ${ }^{1}$ Walter Mendoza, ${ }^{8}$ \\ Daniel Jeannetot, ${ }^{1}$ Daniel Weibel, ${ }^{9}$ Harriet Mayanja-Kizza, ${ }^{10}$ Sanjay Juvekar ${ }^{11}$
}

To cite: Alba S, Verdonck K, Lenglet A, et al. Bridging research integrity and global health epidemiology (BRIDGE) statement: guidelines for good epidemiological practice. BMJ Global Health 2020;5:e003236. doi:10.1136/ bmjgh-2020-003236

Handling editor Seye Abimbola

Received 26 June 2020

Revised 2 September 2020 Accepted 3 September 2020

Check for updates

\section{(C) Author(s) (or their} employer(s)) 2020. Re-use permitted under CC BY-NC. No commercial re-use. See rights and permissions. Published by BMJ.

For numbered affiliations see end of article.

\section{Correspondence to}

Dr Sandra Alba; s.alba@kit.nl

\section{ABSTRACT}

Background Research integrity and research fairness have gained considerable momentum in the past decade and have direct implications for global health epidemiology. Research integrity and research fairness principles should be equally nurtured to produce high-quality impactful research—-but bridging the two can lead to practical and ethical dilemmas. In order to provide practical guidance to researchers and epidemiologist, we set out to develop good epidemiological practice guidelines specifically for global health epidemiology, targeted at stakeholders involved in the commissioning, conduct, appraisal and publication of global health research. Methods We developed preliminary guidelines based on targeted online searches on existing best practices for epidemiological studies and sought to align these with key elements of global health research and research fairness. We validated these guidelines through a Delphi consultation study, to reach a consensus among a wide representation of stakeholders.

Results A total of 45 experts provided input on the first round of e-Delphi consultation and 40 in the second. Respondents covered a range of organisations (including for example academia, ministries, NGOs, research funders, technical agencies) involved in epidemiological studies from countries around the world (Europe: 19; Africa: 10; North America: 7; Asia: 5; South-America: 3 Australia: 1). A selection of eight experts were invited for a face-to-face meeting. The final guidelines consist of a set of 6 standards and 42 accompanying criteria including study preparation, protocol development, data collection, data management, data analysis, dissemination and communication.

Conclusion While guidelines will not by themselves guard global health from questionable and unfair research practices, they are certainly part of a concerted effort to ensure not only mutual accountability between individual researchers, their institutions and their funders but most importantly their joint accountability towards the communities they study and society at large.

\section{INTRODUCTION}

Bora kujenga daraja kuliko ukuta Better build bridges than walls (Kiswhahili proverb)

\section{Key questions}

What is already known?

- Research integrity and research fairness have gained considerable momentum in the past decade and have direct implications for global health epidemiology.

- Balancing research integrity with the realities of conducting fair global health epidemiological research can be challenging.

- Unfortunately, existing good epidemiological practice guidelines developed by national epidemiological associations are not tailored to the idiosyncrasies of global health and lack international legitimacy.

- Also, existing guidelines for research fairness are not specific to epidemiology.

\section{What are the new findings?}

- Through a Delphi consultation study involving a wide range of experts with experience and expertise in global health research and epidemiology, we developed guidelines for good epidemiological practice in global health that address the core principles of research integrity and fair global health research.

- The final guidelines consist of 6 standards and 42 accompanying criteria including study preparation, study protocol and ethical review, data collection, data management, analysis, reporting and dissemination.

Global health epidemiology studies the causes and consequences of morbidity and mortality across geographical boundaries, with emphasis on equitable disease control and health promotion in low-income and middle-income countries. Research integrity and research fairness have gained considerable momentum in the past decade and have direct implications for global health epidemiology. In this article, we argue that research integrity and research fairness principles should be equally nurtured by global 


\section{Key questions}

What do the new findings imply?

- Guidelines will not by themselves guard global health from questionable and unfair research practices.

- However, guidelines are certainly part of a concerted effort to ensure the accountability of funders, institutions and researchers towards the communities they study and society at large.

- We invite all stakeholders involved in the commissioning, conduct, appraisal and publication of global health research to consider the use of these guidelines in their research.

health epidemiologists who aim to produce high-quality impactful research-but bridging the two can lead to practical and ethical dilemmas. In the light of these reflections, we propose guidelines for epidemiological studies targeted at stakeholders involved in the commissioning, conduct, appraisal and publication of global health research.

Research integrity has emerged as a response to the 'reproducibility crisis' (the inability to reproduce research findings), which has shaken the foundations of most scientific disciplines. ${ }^{1}$ Within epidemiological research findings obtained from ill-designed, badly implemented, inappropriately analysed or selectively reported studies will also lead to irreproducible results. ${ }^{2-4}$ Data fabrication, falsification and plagiarism represent the most extreme case of scientific misconduct and consequently inability to reproduce research findings. Yet, practices in the grey zone between this type of deliberate misconduct and ideal scientific behaviour-denoted as "questionable research practices, ${ }^{5}$ or 'research waste ${ }^{6}$-may be more prevalent and ultimately, more damaging. ${ }^{7}$ The reproducibility crisis can be partly attributed to the limits of hypothesis testing paradigms, ${ }^{389}$ but lack of scientific rigour is also a major contributor. ${ }^{71011}$ A number of scientific regulatory bodies have issued documents over the past decade to foster research integrity and thereby tackle questionable research practices and research waste, either in the form of codes of conduct for researchers (such as in the European Union $(\mathrm{EU})^{12}$ and in India ${ }^{13}$ ) or guidelines and policies (eg, Tanzania ${ }^{14}$ and Uganda ${ }^{15}$ ).

Definitions of research integrity centre around four principles: reliability, honest, respect and accountability. ${ }^{12}$ Efforts to foster research integrity in the EU have resulted in a push for open science in all scientific areas. ${ }^{16}$ In epidemiology, more specifically a number of guidelines for good epidemiological practice have also been developed, such as in Switzerland, ${ }^{17}$ Germany, ${ }^{18}$ the Netherlands ${ }^{19}$ and France. ${ }^{20}$ Yet, as we argue in following paragraphs, global health epidemiologists also need to reckon with the idiosyncrasies of conducting global health research, ${ }^{21}$ which derive from its multidisciplinary nature and emphasis on transnational issues and equity at population level. ${ }^{22}$

Multidisciplinary methods are essential in global health research as most issues' studies are embedded in complex systems centred around human behaviour. The study of complex systems in turn is closely related to reproducibility: even when methods have been implemented properly, contextual factors have a key role and can thereby affect reproducibility of findings. The biomedical focus on finding 'what works' means that experimental designs are considered the gold standard to evaluate public health interventions, ${ }^{23}$ and reproducibility is often considered purely in terms of statistical uncertainty. But as critics of counterfactual methodologies in development have argued, these methods produce very context-specific evidence ('did it work there and then'), which risks overlooking specific sociopolitical and cultural contexts. ${ }^{23}{ }^{24}$ Social sciences (eg, anthropology, sociology, political economy) are uniquely placed to understand the social conditions related to the success (or failure) of interventions and provide essential information about reproducibility to complement statistical uncertainty. This criticism can be extended to the use of experimental designs in global health and means that in order to produce useful evidence to decision-makers ${ }^{25}$ global health epidemiologists need to engage with other related disciplines - and most notably social sciences-in multidisciplinary, interdisciplinary or transdisciplinary research. ${ }^{26}$

Global health's emphasis on transnational issues and equity, on the contrary, implies that research integrity needs to be expanded to include research fairness principles. One of the principles of research integrity is respect-defined as 'respect for colleagues, research participants, society, ecosystems, cultural heritage and the environment'. ${ }^{12}$ Strictly speaking, the transnational nature of global health refers to the study of determinants and solutions that cross national boundaries, such as climate change or urbanisation. ${ }^{22}$ But in practice-for a host of historical reasons ${ }^{27}$ - transnational research often implies transnational research collaborations and more specifically partnerships between institutions in higher and lower income countries. The power imbalances potentially arising in such partnerships are at the centre of research fairness concerns ${ }^{28-32}$ and are broadly aligned with calls to decolonise global health. ${ }^{33-36}$ More specifically, research fairness aims at redressing some of the power imbalances in global health, which prevent local stakeholders from shaping the research agenda and competing on a level playing field in scientific arenas. In doing so, research fairness seeks to maximise the positive impact of global health research both on local researchers and on local communities. As such, research fairness lies at the core of the principle of respect in global health partnerships.

A broad understanding of research integrity that encompasses research fairness is therefore key to highquality and impactful global health research-yet there is evidence of shortcomings on both fronts currently. While research integrity in global health has not been amply studied, questionable research practices appear to be equally widespread in this field as in any other. ${ }^{21}$ Many 
different factors can lead to misconduct in research, and global health is not immune to any of these. ${ }^{37} 38$ Also, two recent studies have shown that research fairness-or lack thereof-plays a central role in global health collaborations between researchers in both high-income and low-income countries ${ }^{39}$ and can lead to high levels of moral distress. ${ }^{40}$ Reported challenges included failure to recognise the scientific merit of local staff, the absence of local benefits for the populations studied, ${ }^{39}$ inequitable allocation of public health resources, conflicts of interest, lack of autonomy among community members or beneficiaries of programmes and inadequate access to essential services. ${ }^{40}$

In our experience, balancing research integrity with the realities of conducting fair global health epidemiological research can be challenging. Unfortunately, existing good epidemiological practice guidelines developed by national epidemiological associations ${ }^{17-20}$ are not tailored to the idiosyncrasies of global health and lack international legitimacy. Also, existing guidelines for research fairness are not specific to epidemiology. ${ }^{283041} 42$ In table 1 , we describe potential tensions that can arise in

Table 1 Potential tensions reconciling research integrity and research fairness principles in global health epidemiological practice

\section{Study phase}

\begin{tabular}{|c|c|}
\hline Study preparation & $\begin{array}{l}\text { Research integrity emphasises the establishment of study groups and constructing meaningful } \\
\text { research questions based on a systematic review of the literature. }{ }^{17-20} \text { In addition in global health: } \\
\text { Research fairness implies a need for engagement with key local stakeholders to ensure that } \\
\text { research is driven national public health and research priorities }{ }^{67} \text { (not only those of the higher } \\
\text { income country parties). } \\
\text { - Research fairness also implies a need to ensure that collaborative research furthers local research } \\
\text { systems and competitiveness }{ }^{55} \text { (not only those of the higher income country parties). }\end{array}$ \\
\hline Protocol development & $\begin{array}{l}\text { Research integrity emphasises the need for a detailed study protocol including, which should ideally } \\
\text { be made public, and the need for successful ethical review before starting data collection. }{ }^{17-20} \text { In } \\
\text { addition in global health: } \\
\text { Transnational research implies complications can arise when multiple reviews are required, }{ }^{45} \\
\text { especially if review is not possible at one site (for lack of institutional resources or willingess) or if } \\
\text { reviews conflict with each other. }\end{array}$ \\
\hline
\end{tabular}

Data collection

Research integrity emphasises that studies should be carried out in accordance with the study protocol. Protocol deviations should be recorded, quality checks should be included and copies of the data collected should be stored in secure places. Participants should be well informed about the study and their rights. ${ }^{17-20}$ In addition in global health:

- Transnational research implies potentially harmful effects of data collection for the community as external researchers (non-national or, eg, from different socioeconomic, religious, ethnic background) may cause health, cultural or social or economic harm through the manner in which the conduct research.

- Equity and population-level research implies re-analysis of nationally representative surveys and routine health information systems data. ${ }^{689}$

- Research fairness principles imply that conditions for use and publication should be clearly and fairly negotiated with data owners.

Data management $\quad$ Research integrity emphasises the need for reproducible and traceable procedures. ${ }^{17-20}$ However, in global health:

- Transnational research implies complications can arise due to poor accessibility of study sites and difficult communication when team members are geographically spread out or the conditions are unique to the place where the investigation is carried out.

$\begin{array}{ll}\text { Data analysis } & \text { Research integrity emphasises that statistical analysis should be conducted according to the } \\ \text { protocol. }{ }^{17-20} \text { Additional unforeseen analyses should be clearly justified. However in global health: } \\ \text { Multidisciplinary research implies that it can be difficult to specify statistical analysis plans at the } \\ \text { outset, as methods are often adaptive with quantitative analyses informing the qualitative analyses } \\ \text { or vice versa. }{ }^{70}\end{array}$

Dissemination and communication
Research integrity emphasises scientists' responsibility to report study results in the form of scientific publications. ${ }^{17-20}$ Public data sharing is encouraged because reuse of data makes research more useful and cost-effective. ${ }^{71}$ However, in global health:

- Research fairness means that data sharing should not turn into an unfair one-way process providing valuable data for scientists in high-income countries who may not have contributed to study design and data collection. ${ }^{72}$

- Research fairness also implies the use of methods to ensure effective feedback to affected communities by means of tailored messages and appropriate means of communication. ${ }^{42}$ 
Preliminary guidelines

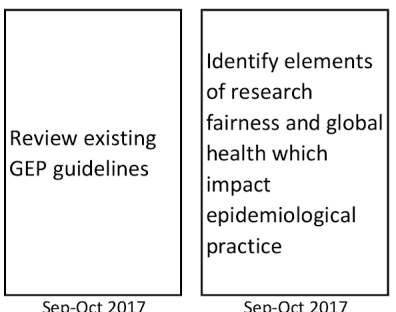

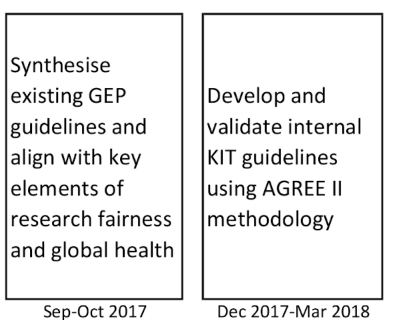

Validated guidelines

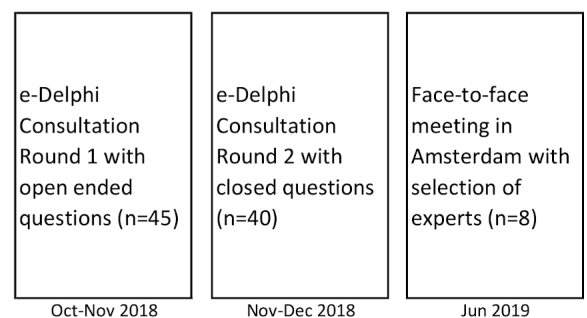

Figure 1 Overview of guideline development steps. GEP, good epidemiological practice.

each of the study phase when trying to align the principles of research integrity and research fairness within the frame of global health practice, by referring to the four main tenets of global health research described above: multidisciplinary, transnational, equity focused and at population-level.

Against this backdrop, we embarked on a study to develop guidelines for good epidemiological practice in global health targeted at stakeholders involved in the commissioning, conduct, appraisal and publication of global health research. The aim of this study was to develop guidelines in which the principles of research integrity are applied while at the same time acknowledging the needs and realities of conducting fair global health research.

\section{METHODS}

\section{Preliminary guidelines}

Between October 2017 and March 2018, we conducted a literature review and developed a preliminary set of guidelines for internal use at KIT Royal Tropical Institute, following the AGREE II methodology. ${ }^{43}$ The development consisted of four steps (figure 1).

First, we performed online searches in Google and PubMed to gather information on existing best practices for epidemiological studies by combining the word 'epidemiology' or 'epidemiological' with the following keywords: 'guidelines', 'guidance', 'procedures', 'standards', 'practice', without any time period restrictions. Results from this search were critically appraised for relevance. In addition, we selected relevant reporting guidelines from the EQUATOR network website. ${ }^{44}$ The records identified through this search could be classified into the following categories: (1) ethics guidelines for research ${ }^{45-48}$; (2) guidelines for good epidemiological practice laid out by international ${ }^{49}$ and national epidemiological associations ${ }^{17-20}$ and (3) academic reporting guidelines. ${ }^{50-54}$

Second, we identified key elements of research fairness $^{32} 4255$ and global health ${ }^{22}$ that have an impact on epidemiological practice. These have been described in more detail elsewhere ${ }^{21}$ and consist of (1) stakeholder involvement; (2) fair and equal partnerships; (3) multiple ethical reviews; (4) emergency research; (5) equity, gender and intersectionality; (6) multidisciplinary research; (7) secondary analyses of existing data; (8) fair data sharing.

Third, we synthesised existing good epidemiological practice guidelines from our literature search and adapted them in order to ensure an alignment with key elements of global health and research fairness. The output of this exercise was a set of standards and criteria. We opted for the following widely used (though not unanimously agreed on ${ }^{56}$ ) convention: standards are qualitative descriptions of the overall quality expectation for the given step in the process; each standard has a number of corresponding criteria, which constitute a measurable checklist to assess whether the standard has been met.

Lastly, these guidelines were internally validated at KIT by means of three consultative sessions. The first session focused on study preparation, protocol development and ethical review; the second on quality assurance, data collection and data management; and the third on data analysis, reporting dissemination and data storage. A multidisciplinary group of KIT global health researchers and advisors participated in these sessions, including four epidemiologists, five public health doctors and two social scientists. Two external expert reviewers (an epidemiologist from Nigeria and one from Bangladesh) also provided feedback on these guidelines.

\section{Validated guidelines: Delphi study}

We sought external validation of the KIT guidelines by means of a Delphi study, as recommended in the literature on guideline development. ${ }^{5758}$ The Delphi method is a structured iterative process to incorporate the views of a group of experts on a draft product in order to develop a final version that represents agreement by all participants. For the development of guidelines, the Delphi method consists of repeated surveys with participants to assess the level of agreement with each element (standards and criteria) of the guidelines and to collect suggestions for change. The respondents' comments are used to formulate a refined version of the guidelines, which is then submitted for a next round of feedback until a satisfactory level of agreement is reached. ${ }^{58-60}$

The Delphi study was designed in three stages (figure 1): two online 'e-Delphi' consultations ${ }^{61}$ with a larger group of experts and one face-to-face meeting with a selected group of respondents. We conducted a stakeholder analysis $^{62}$ to select a diverse group of experts representing 
the major organisations involved in the commissioning, conduct, appraisal and publication of global health research. As part of this analysis, we considered each stakeholder's interest and position towards good epidemiological practice and their influence on the implementation of studies within and beyond their organisation. We identified and contacted individuals working or affiliated with these organisations through KIT's professional network. To reflect the multidisciplinary and transnational nature of global health research, we aimed to identify experts from a wide range of disciplines and countries.

The tools for online consultation consisted of online SurveyMonkey questionnaires. Before sending tools to study participants, we pilot-tested the tools by requesting KIT epidemiologists who were not involved in this study to complete the survey.

The first round of online consultations was conducted in October and November 2018. This round consisted of open-ended questions to encourage qualitative input from experts. Participants were presented with the guidelines through an online questionnaire and were asked to indicate whether each standard and criterion should be kept, deleted or revised. There was also room for additional comments per standard/criterion as well as comments pertaining to the guidelines in general.

The second round of consultations was conducted in November and December 2018. For transparency and clarity, participants received a document describing the old and revised guidelines including comments on the changes and reformulations that were made. This round was more quantitative in nature. Per standard and criterion, participants were asked to rate whether they agreed with the revised formulations using a 5-point Likert scale (strongly agree to strongly disagree), with opportunities for general comments. The data from this round was analysed by calculating the percentage agreement for each standard and criterion from the Likert scales. Following guidance from Hasson et al, ${ }^{59}$ we did not set an a priori cut-off value for consensus, as there is no universally agreed value.

Finally, a face-to-face meeting was organised in Amsterdam in June 2019 with a selection of experts who had participated in the online consultations. The aim of this meeting was to take stock of the online consultation responses, to resolve issues that remained thorny despite two rounds of consultation and to agree on the final guidelines. Consensus was reached through iterative rounds of group discussions leading to proposals which were tested in plenary discussions. Where needed, proposal amendments were discussed and further tested for agreement until a satisfactory level of acceptance from all participants was reached.

\section{Ethical review}

Prior to conducting the Delphi consultation, the KIT Royal Tropical Institute's Research Ethics Committee was approached to review the study's tools and protocols. The study was exempted from full ethical review

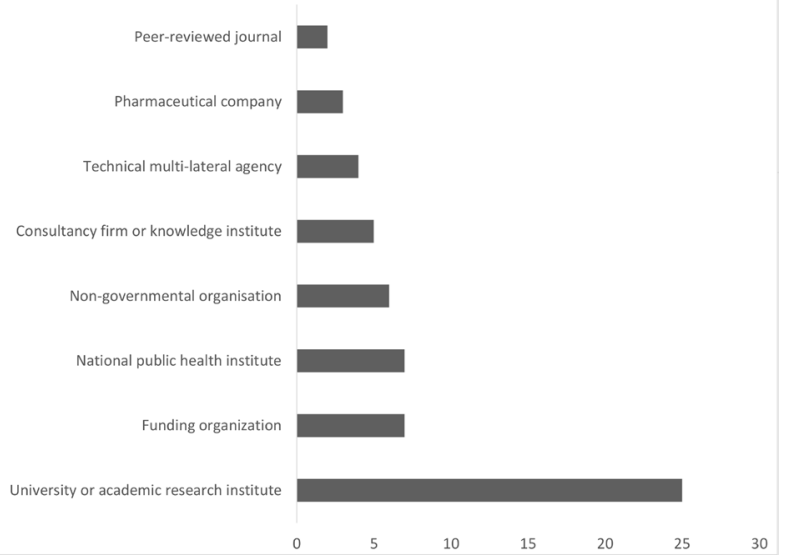

Figure 2 Number of e-Delphi round 1 respondents by type of organisation ( $\mathrm{N}=45)$ [1]. [1]One individual can belong to more than one type of organisation.

on the following grounds: (1) data collection is by means of voluntary survey, which does not contain any personal questions and only covers information related to respondents' duties; (2) since it is an online survey, participants can decline or withdraw participation at any moment without this having any consequences. While we asked participants for their names and affiliations, this was not compulsory.

\section{Participants and public involvement}

Participants received a draft version of this article prior to its submission and the final article will be shared on publication. We considered this an appropriate means of dissemination since all participants are closely involved in academic research. In addition, the website bridge-statement.org will include additional dissemination material, such as translations of this article (in French, Spanish) and training material for epidemiology and global health postgraduate courses.

\section{RESULTS \\ Participants}

We invited 163 people by email to contribute to the first round of the Delphi validation exercise. Forty-five (28\%) participants agreed to participate and completed the first round. Participants in the first round represented various disciplines and organisations (figure 2) and came from a wide range of countries (figure 3 ). Forty experts participated in the second round. Despite the drop in participants, we were able to keep the same balance of geographical and disciplinary representation in both rounds. Finally, for the face-to-face meeting in Amsterdam, we selected eight experts who had participated in the online consultations. These were chosen based on their professional and geographical background, as well as their input in the previous rounds. We therefore invited four delegates from the Netherlands and Belgium, and four international delegates from India, Tanzania, Uganda and Peru. 


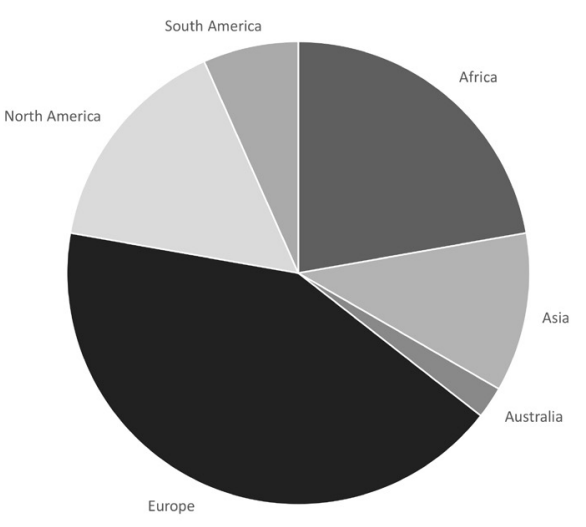

Figure 3 Number of e-Delphi Round 1 respondents by region $(\mathrm{N}=45)$ [1]. [1]Refers to individuals' working location, which is at times different from their country of origin.

\section{Revision of guidelines}

The preliminary guidelines consisted of 51 criteria across nine standards covering the following nine implementation steps for the conduct of an epidemiological study : (1) study preparation; (2) study protocol and ethical review; (3) quality assurance; (4) statistical analysis plan; (5) data collection; (6) data management; (7) analysis; (8) reporting and dissemination; (9) data storage.

Overall, the e-Delphi consultation led to a substantial simplification and shortening of the guidelines. After the first round of online consultations, 14 criteria out of the original 51 were deleted $(27 \%), 5$ were added, and all others were reworded. The statistical analysis and quality assurance standards were removed as they were considered too detailed and prescriptive. In their comments, participants warned against the risk of having too many standards and criteria as well as overlap with other existing guidelines. Furthermore, the data storage standard was dismantled and criteria were placed in the data management and dissemination standards. We revised the criteria that were considered important but not always attainable or applicable, with wording such as 'strive for' and 'consider what is in the remit' to ensure they were reflected but not considered mandatory.

A total of 6 standards and 45 criteria were taken into the second round of online consultations. Overall, there was a high level of agreement in the second round, with all but two criteria achieving at least $85 \%$ of the 40 respondents grading as 'strongly agree' or 'agree' that the standards/criteria should be part of the final guidelines in their revised formulation. The criterion with the highest disagreement was the data analysis criteria 'Analysts should not work with data that have direct personal identifiers' (undecided: 24\%; disagree: $8 \%$; strongly disagree: $3 \%$ ). The main dissatisfaction was that direct identifiers might be date of birth or a postcode and may be needed for analyses. Instead, respondents favoured a focus on a limited number of people handing personal data, alongside training for data handlers on confidentiality and data security. The criterion with the second highest disagreement was the protocol development criterion: 'The protocol should include a clear and complete data statistical analysis plan which excludes data-driven adaptations' (undecided: 11\%; disagree: $8 \%$ ). This criterion was considered unclear, rigid, unrealistic and potentially leading to inadequate analysis strategies. Instead, respondents favoured a stronger focus on transparency in the reporting stage.

During the face-to-face meeting in Amsterdam, we further streamlined and simplified the standards and criteria taking into account the qualitative feedback from all survey respondents. The outcome of the overall study consists of a final set of 6 standards and 42 accompanying criteria covering the following steps in the study process: (1) study preparation; (2) study protocol and ethical review; (3) data collection; (4) data management; (5) analysis; (6) dissemination and communication (table 2). A glossary of terms can be found in an accompanying explanation and elaboration paper (REF). ${ }^{63}$

\section{DISCUSSION}

Based on literature and expert opinion, we have compiled a set of standards for good epidemiological practice in global health, bridging research integrity and research fairness. While there is an inherent tension between efforts to conduct research according to strict technical guidelines of good epidemiological practice and the realities of conducting global health research, we believe these guidelines offer a practical support to epidemiologists to navigate through the complex global health landscape.

Our aim was not to duplicate existing work, but rather to bring together existing principles in one overarching guideline with a focus on practical implications for researchers. While this article has focused on the justification and methodology followed for the development of the guidelines, further explanation and elaboration on all standards and criteria can be found in the accompanying publication (REF) ${ }^{63}$ Wherever possible in our explanation and elaboration document, we have made reference to existing tools and guidelines.

There are a number of limitations to our study with a potential impact on the resulting guidelines. First, these guidelines are based on a literature review that was conducted in 2017. While we made all attempts to supplement this review with relevant literature that we encountered thereafter, it is possible that we omitted important recent developments in the field of global health epidemiology and research fairness. Second, our recommendations are very much influenced by the opinions and experience of participants of the Delphi consensus study, and more specifically the experts which participated in the face-to-face meeting. While we made all attempts to ensure a diverse group of expertise and geographical background reflecting the target group for these guidelines, it is possible that some perspectives may not have been given duly prioritised. Third, through the e-Delphi 
Table 2 Final BRIDGE checklist

\# Standard 1. Study preparation: carefully prepare the study, in partnership with local researchers, by taking into account existing knowledge and resources and engaging with key stakeholders

1.1 Plan and execute research in partnership with local researchers. When working in a setting where relevant epidemiological competences are limited or not available, consider what is in the study team's remit to strengthen local capacity

1.2 Identify and engage key stakeholders throughout the study with approaches based on their needs, competences and expectations. Key stakeholders include representatives of affected populations and end-users of research

1.3 Establish the knowledge gap by searching the literature (peer-reviewed publications and grey literature) as well as by consulting (local) experts, representatives of affected populations and end-users

$1.4 \quad$ Develop research questions and objectives in consultation with research partners and expected end-users

1.5 Select study design and research methods to best fulfil the study objectives and give due consideration to multidisciplinary approaches

1.6 Before embarking on primary data collection, assess whether existing data could be used, fully or partly, to fulfil the research objectives

1.7 Ensure data ownership and publication agreements have been agreed by all research partners

1.8 Agree on work plans and governance structures with all study partners. Allocate adequate time, financial and human resources to all phases of the study

\# Standard 2. Protocol development: prepare a detailed research protocol and ensure it has been approved by relevant ethical review boards if it includes research concerning human participants

2.1 Prepare a detailed research protocol in consultation with all research partners

2.2 Write a clear and comprehensive analysis section

2.3 Consider studying the effect of locally relevant equity dimensions

2.4 When conducting multidisciplinary research, describe the purpose and strategies to integrate different analytical methods in the protocol

2.5 Strive to make study protocols publicly available, either on a publicly accessible website or in appropriate study registers

2.6 For all data collection and data use concerning human subjects, obtain ethical approval (or a waiver) ideally from all institutions and countries involved in the protocol. In case of multiple review and disagreement, the review of the country where the data are collected should take precedence

2.7 When working in a setting without ethical review boards or review boards with limited epidemiological capacity, consider what is in the study team's remit to strengthen their epidemiological capacity

2.8 Explicitly state any open data access in the protocol submitted for ethical review and in the informed consent documents

\# Standard 3. Data collection: use valid and reliable instruments and reproducible methods while ensuring culturally appropriate procedures

3.1 Use valid and reliable research instruments

3.2 Ensure that research instruments are locally adapted and culturally appropriate

3.3 Provide concrete guidance for data collection in a document that is available to all data collection staff

3.4 Select data collection staff according to technical as well as cultural criteria. Clarify the roles and responsibilities for each person involved and provide adequate training and support

3.5 Pilot-test and, if possible, field-test all research instruments prior to the start of effective data collection

3.6 Collect data a respectful and safe manner and in an environment which safeguards the confidentiality of respondents

3.7 Put in place quality assurance and control mechanisms to ensure data accuracy, completeness and coherence

\# Standard 4. Data management: manage data with reproducible procedures and ensure compliance with relevant data protection rules

4.1 Put in place data management procedures before effective start of data collection and provide concrete guidance in a document available to all data management staff

4.2 Create and pretest a data entry application prior to effect start of data collection

4.3 Describe all variables in a codebook and consider preparing additional metadata documentation

4.4 Put in place quality assurance and control mechanisms to ensure data accuracy, completeness and coherence

Continued 


\begin{tabular}{|c|c|}
\hline 4.5 & $\begin{array}{l}\text { Annotate all data cleaning and processing steps and strive for reproducibility by means of stored programming } \\
\text { code }\end{array}$ \\
\hline 4.6 & $\begin{array}{l}\text { For each data file define levels of anonymisation and privacy protection as well as corresponding access rights in } \\
\text { line with national and international frameworks }\end{array}$ \\
\hline 4.7 & $\begin{array}{l}\text { At the beginning of the study, prepare an electronic secured study file to store all study documentation and } \\
\text { outputs. Regularly update this file and archive it the end of the study }\end{array}$ \\
\hline 4.8 & $\begin{array}{l}\text { Retain source data safely, in their original form, preserving data confidentiality for as long as has been described } \\
\text { in the protocol }\end{array}$ \\
\hline \# & $\begin{array}{l}\text { Standard 5. Data analyses: analyse data according to the protocol and integrate statistical analyses with } \\
\text { approaches from other disciplines in the study }\end{array}$ \\
\hline 5.1 & Only work with personal identifiers that are necessary to answer the research questions \\
\hline 5.2 & $\begin{array}{l}\text { Conduct statistical analyses in accordance with the protocol and distinguish preplanned from exploratory } \\
\text { analyses }\end{array}$ \\
\hline 5.3 & Fully annotate all analysis steps and strive for reproducibility by means of programming code \\
\hline 5.4 & $\begin{array}{l}\text { In multidisciplinary studies, integrate statistical analyses with analyses from other study disciplines in an iterative } \\
\text { process to coherently address the research objectives }\end{array}$ \\
\hline 5.5 & Put in place quality assurance and quality control mechanisms to ensure that data has been correctly analysed \\
\hline \# & $\begin{array}{l}\text { Standard 6. Dissemination and communication: report and disseminate results, preferably in the public } \\
\text { domain, with means of communication which appropriately target key stakeholders }\end{array}$ \\
\hline 6.1 & $\begin{array}{l}\text { Develop user-specific dissemination and communication plans in consultation with key stakeholders } \\
\text { (representatives of the affected populations and end-users) }\end{array}$ \\
\hline 6.2 & Report data in a non-stigmatising, non-discriminatory, culturally sensitive and non-identifying manner \\
\hline 6.3 & Conform to reporting guidelines for the given study design and methods in academic publications \\
\hline 6.4 & $\begin{array}{l}\text { Put in place quality assurance and quality control mechanisms to ensure complete, accurate, accessible and } \\
\text { interpretable data reporting }\end{array}$ \\
\hline 6.5 & Consider indexed open access journals for scientific publications \\
\hline 6.6 & $\begin{array}{l}\text { On study completion, consider publication of the archive in an openly accessible online repository. Consult key } \\
\text { stakeholders and research partners to identify strategies within the study team's remit to encourage as much as } \\
\text { possible reanalyses by local researchers }\end{array}$ \\
\hline
\end{tabular}

validation process, the original guidelines lost a number of features which made them very specific to epidemiology (eg, an entire standard on steps involved in the development of statistical analysis plan was dropped). As a result, it could be argued that the current guidelines reflect good research practice more broadly, rather than good epidemiological practice specifically. The accompanying explanation and elaboration notes (REF) do provide further epidemiological details for a number of item of the guidelines.

Nevertheless, we hope that the greatest benefit of these guidelines will be to contribute to current efforts in global health seeking to place communities, societies and researchers from low-income and middle-income countries at the heart of research endeavours in their own countries. With regard to researchers more specifically, we hope these guidelines can improve the quality of epidemiological research by offering explicit recommendations for epidemiologists who are uncertain about how to proceed and challenge the beliefs of researchers accustomed to outmoded practices. ${ }^{64}$ These guidelines may also be considered to support quality improvement activities, by providing the bases for the design of quality assessment (eg, audits) and quality assurance (eg, risk analysis) tools. ${ }^{64}$

We are aware that guideline implementation is a much more challenging process than guidelines development. One of the major hurdles is that guidelines can be seen as inconvenient and time-consuming by practitioners. ${ }^{64}$ In global health research, challenges around implementation are compounded by the great heterogeneity in study contexts and large number of involved parties, ${ }^{37} 38$ resulting in a blurring of responsibilities and conflicting incentives and priorities. ${ }^{65}$ Furthermore, epidemiological practice itself varies widely in scope. The sophistication of strategies to ensure their successful completion should be commensurate to their level of complexity. Factors influencing complexity include the type of setting, the number of study participants, the number of study sites, the types and quantity of data collected per participant, the duration of the study, the mix of disciplines, and so on. In this regard, we believe the adaptation of these guideline to suit local contexts and specific institutional practices and process will be key for implementation. Guideline adaptation frameworks provide a systematic way of approaching adaptation, and their use may 
increase transparency, methodological rigour and the quality of the adapted guideline. ${ }^{66}$

Guidelines will not by themselves guard global health from questionable and unfair research practices. But they are certainly part of a concerted effort to ensure the accountability of funders, institutions and researchers towards the communities they study and society at large. The intent of these guidelines is neither to cripple researchers' freedom nor to impose inflexible rules on the conduct of studies-but rather to streamline efforts and stimulate humility and reflection in order to generate high-quality and impactful research. We invite all stakeholders involved in the commissioning, conduct, appraisal and publication of global health research to consider the use of these guidelines in their research. We welcome any feedback at gep@kit.nl. All comments received will be considered as part of our planned quinquennial revision.

\author{
Author affiliations \\ ${ }^{1}$ Health, KIT Royal Tropical Institute, Amsterdam, The Netherlands \\ ${ }^{2}$ Tropical Diseases, Institute of Tropical Medicine, Antwerp, Belgium \\ ${ }^{3}$ Médecins Sans Frontières, Amsterdam, The Netherlands \\ ${ }^{4}$ Department of Medical Microbiology, Radboudumc, Nijmegen, The Netherlands \\ ${ }^{5}$ National Institute for Medical Research, Dar es Salaam, Tanzania, United Republic \\ of \\ ${ }^{6}$ Big Data Institute, University of Oxford, Oxford, UK \\ ${ }^{7}$ NWO-WOTRO Science for Global Development, The Hague, The Netherlands \\ ${ }^{8}$ United Nations Population Fund, Lima, Peru \\ ${ }^{9}$ EDCTP, The Hague, The Netherlands \\ ${ }^{10}$ School of Medicine, Makerere University, Kampala, Uganda \\ ${ }^{11}$ Vadu Rural Health Program, KEM Hospital Research Centre, Pune, India
}

Acknowledgements This work was made possible thanks to generosity of many people who selflessly gave their time and shared their insights and knowledge throughout all steps of this study. Heartfelt thanks to Christian Schindler, Stella van Beers and Paul Klatser, Fernando Maldonado, Mirjam Bakker, Lisanne Gerstel and Lindy van Vliet for providing the space for initial reflections on these themes including in the post-graduate training programs of both Swiss TPH and KIT Royal Tropical Institute. We are also very grateful to Gerard Swaen for encouraging us to embark on an external validation study and to Prisca Zwanikken for coaching us on the necessary steps. Special thanks to Christina Mergenthaler who played a key role in drafting the first internal KIT guidelines and many thanks to KIT colleagues who provided critical and thoughtful feedback in the internal consultations sessions: Ente Rood, Lucie Blok, Maaike Flinkenflögel, Barend Gerretsen, Egbert Sondorp, Pam Baatsen, Irene de Vries, Tasneem Kakal, Ingrid Zuleta, Ankie van den Broek, Margo van Gurp, Frouwke Veenstra, Eelco Jacobs. We are indebted to Hassan Abiodun and Sayera Banu for their critical expert external review of the internal KIT guidelines. Thanks to Ewen Le Borgne for great suggestions on how to conduct the face-to-face consensus meeting in Amsterdam. Last but not least, our deep appreciation goes to all the e-Delphi participants for their time and thoughtful input: Seye Abimbola, Brenda Kateera, Frode Forland, Solomon Narh-Bana, Carmen Varela Santos, Lex Bouter, Jennita Reefhuis, Suzanne Verver, Antonio Barnabe Ortiz, Sandro Colombo, Cudjoe Bennet, Maya Subelj, Tracy Glass, Susan van den Hof, Borna Mueller, Michiel de Boer, Miguel Antonio Trelles Centurion.

Contributors SA conceived the study, led its execution and compiled the first draft of this manuscript. IT coordinated the implementation of the online Delphi consultation study and wrote the methodology description. MS participated in the consultation sessions for the development of the initial guidelines and DJ and MS led the face-to-face expert review meeting. KV, SFR, AL, SJ, DW, HM-K and WM participated in the expert face-to-face meeting and took final decisions with regard to the formulation of the standards and criteria. All authors read and approved the final version of this manuscript.

Funding This study was made possible thanks to funding from the KIT Knowledge Investment Fund

Competing interests None declared.
Patient and public involvement Patients and/or the public were not involved in the design, or conduct, or reporting, or dissemination plans of this research.

Patient consent for publication Not required.

Provenance and peer review Not commissioned; externally peer reviewed.

Data availability statement Data are available in a public, open access repository. 10.5281/zenodo.3903146.

Open access This is an open access article distributed in accordance with the Creative Commons Attribution Non Commercial (CC BY-NC 4.0) license, which permits others to distribute, remix, adapt, build upon this work non-commercially, and license their derivative works on different terms, provided the original work is properly cited, appropriate credit is given, any changes made indicated, and the use is non-commercial. See: http://creativecommons.org/licenses/by-nc/4.0/.

\section{ORCID iD}

Sandra Alba http://orcid.org/0000-0003-2435-624X

\section{REFERENCES}

1 nature. Challenges in irreproducible research, 2020. Available: https://www.nature.com/collections/prbfkwmwvz

2 Altman DG. The scandal of poor medical research. BMJ 1994;308:283-4.

3 loannidis JPA. Why most published research findings are false. PLoS Med 2005;2:e124.

4 Nosek BA, Bishop DVM, Munafò MR. A manifesto for reproducible science. Nature Human Behaviour 2020.

5 Steneck $\mathrm{NH}$. Fostering integrity in research: definitions, current knowledge, and future directions. Sci Eng Ethics 2006;12:53-74.

6 Chalmers I, Bracken MB, Djulbegovic B, et al. How to increase value and reduce waste when research priorities are set. Lancet 2014;383:156-65.

7 John LK, Loewenstein G, Prelec D. Measuring the prevalence of questionable research practices with incentives for truth telling. Psychol Sci 2012;23:524-32.

8 Wasserstein RL, Lazar NA. The ASA Statement on $p$-Values: Context, Process, and Purpose. Am Stat 2016;70:129-33.

9 Amrhein V, Korner-Nievergelt F, Roth T. The earth is flat $(p>0.05)$ : significance thresholds and the crisis of unreplicable research. PeerJ 2017;5:e3544.

10 Fanelli D. How many scientists fabricate and falsify research? A systematic review and meta-analysis of survey data. PLoS One 2009;4:e5738.

11 Chan A-W, Song F, Vickers A, et al. Increasing value and reducing waste: addressing inaccessible research. Lancet 2014;383:257-66.

12 ALLEA - All European Academies. The European code of conduct for research integrity. Available: https://ec.europa.eu/research/ participants/data/ref/h2020/other/hi/h2020-ethics_code-of-conduct en.pdf

13 Indian Council of Medical Research. Guidelines on code of conduct for research scientists engaged in field of life sciences. Available: https://main.icmr.nic.in/sites/default/files/guidelines/coe\%20of\% 20 conduct $\% 20$ for $\% 20$ research $\% 20$ scientists $\% 20$ engaged $\% 20$ in $\%$ 20the\%20field\%20of\%20life\%20sciences.pdf

14 National Institute for Medical Research. Research policy, guidelines and regulations, 2015. Available: http://www.nimr.or.tz/wp-content/ uploads/2018/11/NIMR_RESEARCH_POLICY_REGULATIONS_2015. pdf

15 Uganda National Council for Science and Technology. Research registration and clearance policy and guidelines, 2016. Available: https://www.uncst.go.ug/guidelines-and-forms/

16 European Comission. Guidelines to the rules on open access to scientific publications and open access to research data in horizon 2020, 2017. Available: https://ec.europa.eu/research/participants/ data/ref/h2020/grants_manual/hi/oa_pilot/h2020-hi-oa-pilot-guide_ en.pdf

17 Altpeter E, Burnand B, Capkun G, et al. Essentials of good epidemiological practice. Soz Praventivmed 2005;50:12-15.

18 Hoffmann W, Latza U, Baumeister SE, et al. Guidelines and recommendations for ensuring good epidemiological practice (GEP): a guideline developed by the German Society for epidemiology. Eur $J$ Epidemiol 2019;34:301-17.

19 Swaen GMH, Langendam M, Weyler J, et al. Responsible epidemiologic research practice: a guideline developed by a working group of the Netherlands epidemiological Society. J Clin Epidemiol 2018;100:111-9. 
20 Recommendations for professional standards and good epidemiological practices (version France 2007), 2020. Available: https://www.ncbi.nlm.nih.gov/pubmed/18841590

21 Alba S, Mergenthaler C. Lies, damned lies and epidemiology: why global health needs good epidemiological practice guidelines. BMJ Glob Health 2018;3:e001019.

22 Koplan JP, Bond TC, Merson MH, et al. Towards a common definition of global health. Lancet 2009;373:1993-5.

23 Bédécarrats F, Guérin I, Roubaud F. All that glitters is not gold. The political economy of randomized evaluations in development. Dev Change 2019;50:735-62.

24 Peeters Grietens K, Gryseels C, Verschraegen G. Misdirection in the margins of malaria elimination methods. Crit Public Health 2019;29:390-400.

25 Pawson R. The shrinking scope of pragmatic trials: a methodological reflection on their domain of applicability. J Clin Epidemiol 2019;107:71-6.

26 Choi BCK, Pak AWP. Multidisciplinarity, interdisciplinarity and transdisciplinarity in health research, services, education and policy: 1. definitions, objectives, and evidence of effectiveness. Clin Invest Med 2006;29:351-64.

27 Johns Hopkins University Press. A history of global health, 2020. https://jhupbooks.press.jhu.edu/title/history-global-health

28 Research Fairness Initiative. Making research partnerships work for everyone, 2019. Available: http://rfi.cohred.org/

29 Musolino N, Lazdins J, Toohey J, et al. COHRED Fairness index for international collaborative partnerships. Lancet 2015;385:1293-4.

30 Lavery JV, IJsselmuiden C. The Research Fairness Initiative: Filling a critical gap in global research ethics. Gates Open Res 2018;2:58.

31 UK Collaborative on Development SciencesDodson J. Building partnerships of equals the role of funders in equitable and effective international development collaborations. Available: https://www. ukcdr.org.uk/wp-content/uploads/2017/11/Building-Partnerships-ofEquals -REPORT-2.pdf

32 TRUST. Global code of conduct for research in resource poor settings. Available: https://www.globalcodeofconduct.org/wpcontent/uploads/2018/05/Global-Code-of-Conduct-Brochure.pdf

33 Costello A, Zumla A. Moving to research partnerships in developing countries. BMJ 2000;321:827-9.

34 Pai M. Global health still mimics colonial ways: here's how to break the pattern. The Conversation. Available: http://theconversation. com/global-health-still-mimics-colonial-ways-heres-how-to-breakthe-pattern-121951

35 Cáceres CF, Mendoza W. Globalized research and "national science": the case of Peru. Am J Public Health 2009;99:1792-8.

36 Abimbola S. The foreign gaze: authorship in academic global health. BMJ Glob Health 2019;4:e002068.

37 Bonn NA, Pinxten W. Rethinking success, integrity, and culture in research (Part 1) - a multi-actor qualitative study on success in science. bioRxiv 2020

38 Bonn NA, Pinxten W. Rethinking success, integrity, and culture in research (Part 2) - a multi-actor qualitative study on problems of science. bioRxiv 2020.

39 Parker M, Kingori P. Good and bad research collaborations: researchers' views on science and ethics in global health research. PLoS One 2016;11:e0163579.

40 TEPHINET. New survey highlights the need for more ethics training in field epidemiology training programs (FETPs). Available: https:// www.tephinet.org/new-survey-highlights-the-need-for-more-ethicstraining-in-field-epidemiology-training-programs

41 CCGHR|CCRSM. Principles for Global Health Research - Canadian Coalition for Global Health Research. Available: https://www.ccghr. $\mathrm{ca} /$ resources/principles-global-health-research/ [Accessed $20 \mathrm{Dec}$ 2019].

42 Swiss Commission for Research Partnerships with Developing Countries (KFPE). A Guide for Transboundary Research Partnerships - 11 Principles, 2018. Available: https://naturalsciences.ch/service/ publications/9505-a-guide-for-transboundary-research-partnerships3rd-edition-2018-

43 Brouwers MC, Kho ME, Browman GP, et al. Agree II: advancing Guideline development, reporting and evaluation in health care. Can Med Assoc J 2010;182:E839-42.

44 The EQUATOR Network. Enhancing the quality and transparency of health research. Available: https://www.equator-network.org/ [Accessed 11 May 2020].

45 World Health Organization, Council for International Organizations of Medical Sciences. International ethical guidelines for health-related research involving humans. Geneva: CIOMS, 2017.
46 Council for International Organizations of Medical Sciences, World Health Organization. International ethical guidelines for biomedical research involving human subjects. Geneva: CIOMS, 2002: 112 p.

47 Council for International Organizations of Medical Sciences. International ethical guidelines for health-related research involving humans. Geneva: CIOMS, 2017.

48 Gopichandran V, Luyckx VA, Biller-Andorno N, et al. Developing the ethics of implementation research in health. Implement Sci 2016;11:161.

49 Good epidemiological practice (GEP) proper conduct in epidemiologic research. Available: http://alraziuni.edu.ye/ book1/Health\%20and\%20Society/Good\%20Epidemiological\% 20Practice\%20(GEP)\%20Proper\%20Conduct\%20in\% 20Epidemiologic\%20Research.pdf [Accessed 30 Apr 2020].

50 Schulz KF, Altman DG, Moher D, et al. Consort 2010 statement: updated guidelines for reporting parallel group randomised trials. BMJ 2010;340:c332

51 von Elm E, Altman DG, Egger M, et al. The strengthening the reporting of observational studies in epidemiology (STROBE) statement: guidelines for reporting observational studies. J Clin Epidemiol 2008;61:344-9.

52 Chan A-W, Tetzlaff JM, Altman DG, et al. Spirit 2013 statement: defining standard protocol items for clinical trials. Ann Intern Med 2013;158:200-7.

53 Pinnock H, Barwick M, Carpenter CR, et al. Standards for reporting implementation studies (STARI) statement. BMJ 2017;356:i6795.

54 O'cathain A, Murphy E, Nicholl J. The quality of mixed methods studies in health services research. $J$ Health Serv Res Policy 2008;13:92-8.

55 The Research Fairness Initiative. Rfi reporting guide, 2018. Available: http://rfi.cohred.org/wp-content/uploads/2018/04/RFI_ ReportingGuide_EN_v2.0_20180315.pdf

56 Donabedian A. Criteria, norms and standards of quality: what do they mean? Am J Public Health 1981;71:409-12.

57 Moher D, Schulz KF, Simera I, et al. Guidance for developers of health research reporting guidelines. PLoS Med 2010;7:e1000217.

58 Murphy MK, Black NA, Lamping DL, et al. Consensus development methods, and their use in clinical Guideline development. Health Technol Assess 1998;2:i-iv, 1-88.

59 Hasson F, Keeney S, McKenna H. Research guidelines for the Delphi survey technique. J Adv Nurs 2000;32:1008-15.

60 Powell C. The Delphi technique: myths and realities. J Adv Nurs 2003;41:376-82.

61 Gill FJ, Leslie GD, Grech C, et al. Using a web-based survey tool to undertake a Delphi study: application for nurse education research Nurse Educ Today 2013;33:1322-8.

62 Varvasovszky Z, Brugha R. A stakeholder analysis. Health Policy Plan 2000;15:338-45.

63 Alba S, Lenglet A, Verdonck K. Bridging research integrity and global health epidemiology (bridge) guidelines: explanation and elaboration. BMJ Global Health 2020:e003237.

64 Woolf SH, Grol R, Hutchinson A, et al. Clinical guidelines: potential benefits, limitations, and harms of clinical guidelines. $B M$ 1999;318:527-30.

65 Field MJ, Lohr KN. Guidelines for clinical practice: from development to use, 1992. Available: http://www.vlebooks.com/vleweb/product/ openreader?id=none\&isbn=9780309537940 [Accessed 30 Aug 2020].

66 Wang Z, Norris SL, Bero L. The advantages and limitations of guideline adaptation frameworks. Implementation Sci 2018;13:72.

67 World Health Organisation. The who strategy on research for health, 2012. Available: https://www.who.int/phi/WHO Strategy on research_for_health.pdf

68 Victora CG, Black RE, Boerma JT, et al. Measuring impact in the millennium development goal era and beyond: a new approach to large-scale effectiveness evaluations. Lancet 2011;377:85-95.

69 Wagenaar BH, Sherr K, Fernandes Q, et al. Using routine health information systems for well-designed health evaluations in low- and middle-income countries. Health Policy Plan 2016;31:129-35.

70 Creswell JW, Clark VickiLPlano. Designing and conducting mixed methods research, 2017. https://wordery.com/designingand-conducting-mixed-methods-research-john-w-creswell9781506386621

71 Wilkinson MD, Dumontier M, Aalbersberg IJJ, et al. The fair guiding principles for scientific data management and stewardship. Sci Data 2016;3:1-9.

$72 \mathrm{WHO}$. Sharing health data: developing country perspectives. Available: https://www.who.int/bulletin/volumes/88/6/10-079129/en/ [Accessed 29 Apr 2020]. 